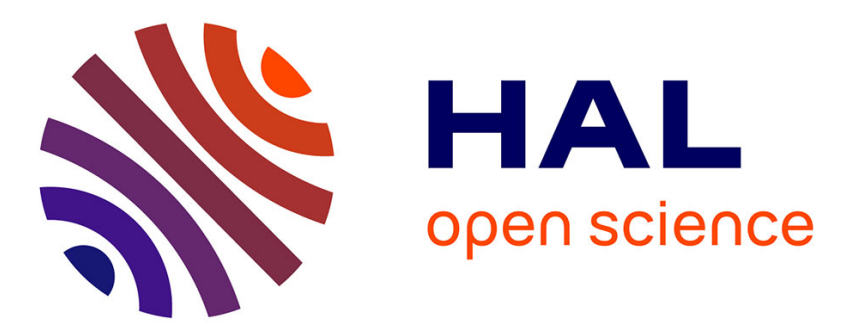

\title{
Consensus of Multi-Agent Systems With Nonholonomic Restrictions via Lyapunov's Direct Method
}

Mohamed Maghenem, Abraham Bautista, Emmanuel Nuno, Antonio Loria, Elena Panteley

\section{- To cite this version:}

Mohamed Maghenem, Abraham Bautista, Emmanuel Nuno, Antonio Loria, Elena Panteley. Consensus of Multi-Agent Systems With Nonholonomic Restrictions via Lyapunov's Direct Method. IEEE Control Systems Letters, 2019, 3 (2), pp.344-349. 10.1109/LCSYS.2018.2879043 . hal-02367702

\section{HAL Id: hal-02367702 \\ https://hal.science/hal-02367702}

Submitted on 5 Mar 2020

HAL is a multi-disciplinary open access archive for the deposit and dissemination of scientific research documents, whether they are published or not. The documents may come from teaching and research institutions in France or abroad, or from public or private research centers.
L'archive ouverte pluridisciplinaire HAL, est destinée au dépôt et à la diffusion de documents scientifiques de niveau recherche, publiés ou non, émanant des établissements d'enseignement et de recherche français ou étrangers, des laboratoires publics ou privés. 


\title{
Consensus of Multi-agent Systems with Nonholonomic Restrictions via Lyapunov's Direct Method
}

\author{
Mohamed Maghenem Abraham Bautista Emmanuel Nuño Antonio Loría Elena Panteley
}

\begin{abstract}
This paper presents a smooth time-varying $\delta$ persistently exciting controller for full consensus of autonomous nonholonomic vehicles modelled as unicycles. This consists in the robots assuming a common prescribed Cartesian position relative to an unknown barycentre and an unknown common orientation. More significantly, for the first time in the literature, a strict Lyapunov function is provided and uniform global asymptotic stability for the closed-loop system is established. This is well beyond weaker convergence properties that are more commonly guaranteed in the literature.
\end{abstract}

\section{INTRODUCTION}

In multi-agent systems, consensus means that the state of each agent reaches an agreement coordinate value that is not, in general, a priori specified as a set-point target [1]. The consensus problem has been extensively studied for networks of linear systems [2], [3], [4] and for different classes of nonlinear systems [5], [6], [7]. Distributed solutions to cooperative control problems including consensus and formation problems have been addressed, for instance, in [8], [9], [2], [1], [10], [11], [12]. However, these results cannot be applied in multiagent systems with nonholonomic constraints.

We distinguish two types of the consensus problems for such vehicles: partial-consensus, in which a reference orientation is imposed to each vehicle and full-consensus, in which it is additionally required that all vehicles assume the same nonpredetermined orientation.

Consensus of nonholonomic autonomous vehicles has been extensively studied in the literature during the last decade. Necessary and sufficient conditions for the feasibility of a class of position formations are formulated in [13]. In [14] a discontinuous decentralized feedback controller that drives a network of nonholonomic unicycles to a rendezvous point in terms of both position and orientation is proposed. Smooth time-varying controllers are also used for distributed formation control. For instance, in [15] a consensus-based approach is used in order to drive a group of agents to a desired geometric pattern; this work is extended in [16] by introducing a PD-based controller for the velocity dynamics; in [17] the position/orientation control problem is addressed that leads the agents to a given formation using only their orientation.

M. Maghenem is with the University of California at Santa Cruz. Email: mmaghene@ucsc.edu. A. Bautista and E. Nuño are with the Dept. of Computer Science CUCEI, at University of Guadalajara, Guadalajara, Mexico. E-mails: abraham.bautista@academicos.udg.mx and emmanuel.nuno@cucei.udg.mx, respectively. A. Loría and E. Panteley are with the CNRS. LSS-CentraleSupelec, 3 Rue Joliot Curie, 91192, France. E-mails loria(panteley)@1ss.supelec.fr. E. Panteley is also with ITMO University, Kronverkskiy av. 49, Saint Petersburg, 197101, Russia.

This article is supported by Dept. STITS of University of Paris Saclay, by the Government of Russian Federation (grant 08-08), by ECOS-Nord contract M14M02, and by the Mexican CONACyT Basic Scientific Research grant CB-282807.
Consensus is also addressed in [18] via a cooperative control law that is robust to constant communication delays. In [19] a distributed control law for a network of nonholonomic agents in the presence of bounded disturbances with unknown dynamics in all inputs channels is presented. In [20] the formation control problem with respect to a prespecified setpoint is studied taking into account loss of connectivity and obstacle avoidance.

In this paper we solve the full-consensus problem via descentralized control. Our controller is smooth time-varying and of the $\delta$-Persistently-Exciting class —see [21], [22], [23]. A significant contribution is to establish uniform global asymptotic stability (UGAS) for the closed-loop system. Contrary to the significantly weaker property of non-uniform convergence, which is more commonly established in the literature on robot control, UGAS guarantees robustness with respect to bounded disturbances (local Input-to-State-Stability). Furthermore, in contrast to most of the previous works where only the kinematics model is considered, we use a model augmented by two integrators, which represent the feedback-linearized velocity dynamics.

To the best of our knowledge, apart from the preliminary conference paper [24] where only the partial-consensus problem is addressed - see also [25], for the first time in the literature we provide a strict and differentiable Lyapunov function for the closed-loop system. This function is based on the Mazenc contstruction method to render Lyapunov functions strict, introduced in [26] — see also [27].

The rest of the paper is organized as follows. In the next section we describe the networked systems' model and we formulate the problem at hand. Our main result is presented in Section III and some simulation results are provided in Section IV. Concluding remarks are presentned in Section V.

\section{MODEL DESCRIPTION AND PROBLEM FORMULATION}

Let us consider a swarm of $N$ autonomous vehicles modeled as unicycles, that is, with kinematics

$$
\begin{aligned}
\dot{x}_{i} & =v_{i} \cos \left(\theta_{i}\right) \\
\dot{y}_{i} & =v_{i} \sin \left(\theta_{i}\right) \\
\dot{\theta}_{i} & =\omega_{i}, \quad i \leq N
\end{aligned}
$$

and dynamics ${ }^{1}$

$$
\begin{aligned}
& \dot{v}_{i}=u_{v i} \\
& \dot{\omega}_{i}=u_{\omega i}
\end{aligned}
$$

\footnotetext{
${ }^{1}$ For simplicity, the dynamics is modeled as a simple integrator. There is little loss of generality in this assumption considering that velocity dynamics models are typically feedback linearizable.
} 
where $\nu_{i}:=\left[\begin{array}{ll}v_{i} & \omega_{i}\end{array}\right]^{\top}, \varsigma_{i}:=\left[\begin{array}{lll}x_{i} & y_{i} & \theta_{i}\end{array}\right]^{\top}$. The variables $x_{i}$ and $y_{i}$ denote Cartesian coordinates of a fixed point on the vehicle relative to a fixed frame, $\theta_{i}$ denotes its orientation with respect to the $X$-axis, and $v_{i}$ and $\omega_{i}$ denote the forward and angular velocities respectively.

It is assumed that for each vehicle its pair of control inputs $\left(u_{v i}, u_{\omega i}\right)$ depend on own local coordinates and time, $\left(\nu_{i}, \varsigma_{i}, t\right)$, but also on the coordinates of the neighbors. From a physical viewpoint, there exists a direct relation between the wheels' input torque and the controls $\left(u_{v i}, u_{\omega i}\right)-c f$. [28]; hence, the latter are easily implementable.

It is also assumed that the interconnection topology of the network is determined by a connected, undirected, and static graph whose connectivity is defined by the Laplacian matrix $L:=\left[\ell_{i j}\right] \in \mathbb{R}^{N \times N}$, where

$$
\ell_{i j}=\left\{\begin{array}{cc}
\sum_{j \in \mathcal{N}_{i}} a_{i j} & i=j \\
-a_{i j} & i \neq j
\end{array}\right.
$$

and $a_{i j} \geq 0 ; a_{i j}>0$ if the $i$ th and $j$ th vehicles communicate with each other and $a_{i j}=0$ otherwise.

Remark 1: By construction, $L$ has a zero row sum, i.e., $L \mathbf{1}_{N}=0$, where $\mathbf{1}_{N} \in \mathbb{R}^{N}$ corresponds to the vector $[1 \cdots 1]^{\top}$. Moreover, since the interconnection graph is connected, undirected and static, $L$ is symmetric, it has a single zero-eigenvalue and the rest of the spectrum of $L$ is positive. Thus, $\operatorname{rank}(L)=N-1$.

Two types of consensus are of interest, namely, the partial and the full consensus problems. In the first case, it is required from each of these vehicles to reach a certain Cartesian position relatively to a barycentre with unknown Cartesian coordinates $z_{c}:=\left[\begin{array}{ll}x_{c} & y_{c}\end{array}\right]^{\top}$ and a given desired orientation, that is, given $\left(\delta_{x i}, \delta_{y i}\right) \in \mathbb{R}^{2}$ and $\theta_{d i} \in \mathbb{R}$, it is required that

$$
\begin{gathered}
\lim _{t \rightarrow \infty} x_{i}(t):=x_{c}+\delta_{x i}, \quad \lim _{t \rightarrow \infty} y_{i}(t):=y_{c}+\delta_{y i}, \\
\lim _{t \rightarrow \infty} \theta_{i}(t):=\theta_{d i}, \quad \forall i \leq N .
\end{gathered}
$$

In the case of full consensus, it is required, in addition to (4), that the vehicles adopt a common non-given orientation $\theta_{c}$, i.e.,

$$
\lim _{t \rightarrow \infty} \theta_{i}(t):=\theta_{c}, \quad \forall i \leq N .
$$

These goals are reached via smooth nonlinear dynamic control and by addressing the more challenging stabilization problems described next.

Definition 1 (Consensus-based formation control problem): For a network of $N$ vehicles with a topology represented by a connected, undirected, and static graph, with Laplacian $L$ as above we define partial consensus as the goal of rendering the manifold

$$
\begin{array}{r}
\mathcal{S}_{p c}:=\left\{\varsigma_{i} \in \mathbb{R}^{3}, i \leq N:\left[x_{i}-\delta_{x_{i}}\right]=x_{c},\left[y_{i}-\delta_{y_{i}}\right]=y_{c},\right. \\
\left.\theta_{i}=\theta_{d i}\right\}
\end{array}
$$

uniformly globally asymptotically stable for a given pair $\left(\delta_{x i}, \delta_{y i}\right) \in \mathbb{R}^{2}$ and given desired orientations $\theta_{d i}$. The fullconsensus problem is defined as the property of rendering the manifold

$\mathcal{S}_{f c}:=\left\{\varsigma_{i} \in \mathbb{R}^{3}, i \leq N:\left[x_{i}-\delta_{x_{i}}\right]=x_{c},\left[y_{i}-\delta_{y_{i}}\right]=y_{c}\right.$,

$$
\left.\theta_{i}=\theta_{c}\right\}
$$

uniformly globally asymptotically stable for some nonpredefined $\theta_{c}$.

To address the problems previously defined we introduce an adequate dynamical model for the interconnected vehicles. Let us start by defining the translated coordinates

$$
z_{i}:=\left[\begin{array}{c}
x_{i}-\delta_{x_{i}} \\
y_{i}-\delta_{y_{i}}
\end{array}\right], \quad z:=\left[z_{1}^{\top} \cdots z_{N}^{\top}\right]^{\top}
$$

as well as $\theta=\left[\theta_{1} \cdots \theta_{N}\right]^{\top} \in \mathbb{R}^{N} ; v=\left[v_{1} \cdots v_{N}\right]^{\top} \in \mathbb{R}^{N}$; $\omega=\left[\omega_{1} \cdots \omega_{N}\right]^{\top} \in \mathbb{R}^{N}, \Phi(\theta)=\operatorname{blockdiag}\left[\phi\left(\theta_{i}\right)\right] \in \mathbb{R}^{2 N \times N}$, with $\phi\left(\theta_{i}\right)=\left[\cos \left(\theta_{i}\right) \sin \left(\theta_{i}\right)\right]^{\top}$, and the control inputs $u_{v}=$ $\left[u_{v 1} \cdots u_{v N}\right]^{\top} \in \mathbb{R}^{N}$ and $u_{\omega}=\left[u_{\omega 1} \cdots u_{\omega N}\right]^{\top} \in \mathbb{R}^{N}$. In terms of these variables the dynamics of the networked system is given by

$$
\begin{aligned}
\dot{z} & =\Phi(\theta) v \\
\dot{v} & =u_{v} \\
\dot{\theta} & =\omega \\
\dot{\omega} & =u_{\omega} .
\end{aligned}
$$

Next, we express the relative Cartesian position errors for each vehicle $i$ communicating with $\mathcal{N}_{i}$ neighbors in the $i$ th coordinates frame and we introduce the consensus errors

$$
\begin{aligned}
e_{i} & =\phi\left(\theta_{i}\right)^{\top} \sum_{j \in \mathcal{N}_{i}} a_{i j}\left(z_{i}-z_{j}\right), \\
s_{i} & =\phi\left(\theta_{i}\right)^{\perp \top} \sum_{j \in \mathcal{N}_{i}} a_{i j}\left(z_{i}-z_{j}\right) .
\end{aligned}
$$

where $\phi\left(\theta_{i}\right)^{\perp}=\left[\sin \left(\theta_{i}\right)-\cos \left(\theta_{i}\right)\right]^{\top}$. In the equivalent vector form $e:=\left[e_{1}^{\top} \cdots e_{N}^{\top}\right]^{\top}$ and $s:=\left[s_{1}^{\top} \cdots s_{N}^{\top}\right]^{\top}$ these satisfy

$$
e=\Phi(\theta)^{\top} \mathcal{L} z, \quad s=\Phi(\theta)^{\perp \top} \mathcal{L} z
$$

where $\mathcal{L}:=L \otimes I_{2}$ and, after the following statement,

$$
\lim _{t \rightarrow \infty} z(t)=1_{N} \otimes z_{c}
$$

is equivalent to $(e, s) \rightarrow(0,0)$.

Lemma 1: Consider the error states $(e, s)$ defined in (9); let $L$ be symmetric, have a single zero-eigenvalue, and let the remainder of its spectrum be strictly positive. Then, $\mathcal{L} z=0 \Leftrightarrow$ $(e, s)=(0,0)$ and, moreover,

$$
\lambda_{2}(L) z^{\top} \mathcal{L} z \leq|e|^{2}+|s|^{2} \leq \lambda_{N}(L) z^{\top} \mathcal{L} z
$$

where $\lambda_{2}(L)$ and $\lambda_{N}(L)$ are the second smallest and the largest eigenvalues of $L$, respectively.

Proof. That $\mathcal{L} z=0 \Leftrightarrow(e, s)=(0,0)$ follows from the fact that the matrix $\left[\Phi(\theta) \Phi(\theta)^{\perp}\right]$ is non singular. To establish the inequalities in (11) we use the identity $|e|^{2}+|s|^{2}=z^{\top} \mathcal{L}^{2} z=$ $z^{\top} \mathcal{L}^{1 / 2} \mathcal{L} \mathcal{L}^{1 / 2} z$. Since $\mathcal{L}^{1 / 2} z$ is orthogonal to the eigenspace associated to the zero eigenvalue of $\mathcal{L}$, we have

$\lambda_{2}(L) z^{\top} \mathcal{L}^{1 / 2} \mathcal{L}^{1 / 2} z \leq z^{\top} \mathcal{L}^{1 / 2} \mathcal{L} \mathcal{L}^{1 / 2} z \leq \lambda_{N}(L) z^{\top} \mathcal{L}^{1 / 2} \mathcal{L}^{1 / 2} z$

so (11) follows.

$\triangleleft$

Lemma 1 enables us to "discard" the kinematics equation (8a) 
and replace it by the dynamic equations of $e$ and $s$. These are computed by differentiating on both sides of (9) to obtain

$$
\begin{aligned}
\dot{e} & =-\bar{\omega} s+\Phi(\theta)^{\top} \mathcal{L} \Phi(\theta) v \\
\dot{s} & =\bar{\omega} e+\Phi(\theta)^{\perp \top} \mathcal{L} \Phi(\theta) v
\end{aligned}
$$

for whose computation we used (8a) and

$$
\dot{\Phi}(\theta)=-\Phi(\theta)^{\perp} \bar{\omega}, \quad \dot{\Phi}(\theta)^{\perp}=\Phi(\theta)^{\perp} \bar{\omega}, \quad \bar{\omega}:=\operatorname{diag}\left[\omega_{i}\right] .
$$

Regarding the orientation angles, we introduce the errors $\tilde{\theta}:=\theta-\theta^{*}$ where $\theta^{*}:=\left[\theta_{1}^{*} \cdots \theta_{N}^{*}\right]$. In the case of full consensus, we define $\theta^{*}:=\mathbf{1}_{N} \theta_{c}$ and for partial consensus we define $\theta_{i}^{*}:=\theta_{d i}$.

Thus, recasted in terms of stabilization theory, consensus, in the sense of Definition 1, is achieved if for the dynamic system (12), (8b)-(8d) the origin $\{(e, s, v, \omega, \tilde{\theta})=(0,0,0,0,0)\}$ is rendered uniformly globally asymptotically stable.

\section{CONTROL DESIGN AND MAIN RESULT}

Our main purpose is to address the full-consensus problem for the torque-controlled unicycle. To put our main result in perspective, however, we start by describing the control approach in the context of the partial-consensus problem, solved in the preliminary conference article [24].

\section{A. Partial consensus}

Let, for the time being, $\theta^{*}:=\theta_{d}$ that is, it is assumed that each vehicle assumes its own orientation.

For the translational dynamics $\dot{v}=u_{v}$ we employ a simple Proportional-Derivative controller akin to the one proposed in [16], that is, let

$$
u_{v}=-K_{d t} v-K_{p t} e,
$$

where $K_{d t}$ and $K_{p t}$ are diagonal positive definite matrices. Then, for the rotational dynamics we propose the following $\delta$-persistently-exciting controller — $c f . \quad$ [21], [29]

$$
u_{\omega}=-K_{d \theta} \omega-K_{p \theta} \tilde{\theta}-p(t) \kappa(s, e)
$$

where $K_{d \theta}$ and $K_{p \theta}$ are diagonal positive definite matrices, $p$ is persistently exciting, i.e., there exist $T$ and $\mu>0$ such that

$$
\int_{t}^{t+T}|p(\tau)| d \tau \geq \mu \quad \forall t \geq 0,
$$

and $\kappa(s, e) \in \mathbb{R}^{N}$ is defined as

$$
\kappa(s, e)=\frac{1}{2}\left[s_{1}^{2}+e_{1}^{2} \cdots s_{N}^{2}+e_{N}^{2}\right]^{\top} .
$$

The closed-loop system that results from Equations (8), (9), (13) and (14), is given by Equations (12) and

$$
\begin{aligned}
\dot{v} & =-K_{d t} v-K_{p t} e \\
\dot{\tilde{\theta}} & =\omega \\
\dot{\omega} & =-K_{d \theta} \omega-K_{p \theta} \tilde{\theta}-p(t) \kappa(s, e)
\end{aligned}
$$

Roughly speaking, the stabilization mechanism is the following. The terms $-K_{d t} v-K_{p t} e$ are designed to steer $v$ and $e$ to zero while Equation (12) corresponds to those of a harmonic oscillator perturbed by the vanishing input $v$. The combined effect of this steering and oscillations produces a "spiraling" motion towards the origin, in the plane $(e, s)-c f$. [30]. The oscillatory effect is induced and maintained by keeping $\omega$ away from zero. This is achieved by the $\delta$-persistently-exciting controller $u_{\omega}$. Indeed, note that the equations (12) are those of a stable second order system with input $-p(t) \kappa(s, e)$, which possesses the so-called $\delta$-persistency-of-excitation property with respect to $(e, s)-$ [31]: for any $\delta>0$ there exist $T$ and $\mu>0$ such that

$$
\left|\left[\begin{array}{ll}
e & s
\end{array}\right]\right| \geq \delta \Longrightarrow \int_{t}^{t+T} \kappa(e, s)|p(\tau)| d \tau \geq \mu \quad \forall t \geq 0 .
$$

Technically, the input $-p(t) \kappa(s, e)$ produces a $\delta$ persistently-exciting output $\omega$ which oscillates as long as the error trajectories $|[e(t) s(t)]|$ are away from the origin. The $\delta$-persistency-of-excitation property of $\omega$ is guaranteed by the property that the output of a strictly proper stable linear system driven by a $\delta$-persistently-exciting input is also $\delta$-persistently-exciting [31]. In [24], for the system system (12), (17) with $p(t)$ and up to its 3 rd derivative bounded and $\dot{p}(t)$ is persistently exciting, it is showed that the origin for the is uniformly globally asymptotically stable and a strict Lyapunov function is provided. Below, we present a solution to the full-consensus problem.

\section{B. Full consensus}

Let $\theta^{*}:=\mathbf{1}_{N} \theta_{c}$, which is unknown. For any continuous function $\varphi: \mathbb{R}_{\geq 0} \rightarrow \mathbb{R}^{n \times m}$ let $|\varphi|_{\infty}:=\sup _{t \geq 0}|\varphi(t)|$, where $|\cdot|$ denotes, depending on the context, the absolute value of scalars, the Euclidean norm of vectors, or the induced norm of matrices.

Assumption 1: There exists $b_{p}>0$ such that $\max \left\{|p|_{\infty},|\dot{p}|_{\infty},|\ddot{p}|_{\infty},\left|p^{(3)}\right|_{\infty}\right\} \leq b_{p}$ and, moreover, $\dot{p}(t)$ is persistently exciting, with excitation parameters $(T, \mu)$.

Let $\bar{q}(t)=\operatorname{diag}\left[q_{i}(t)\right] \in \mathbb{R}^{N \times N}$ be defined dynamically via the differential equation

$$
\bar{q}^{(3)}+K_{\alpha} \ddot{\bar{q}}+K_{I} \dot{\bar{q}}=\dot{p}(t) I_{N},
$$

where $K_{I}$ and $K_{\alpha}$ are diagonal positive definite matrices of appropriate dimension that will appear in the control design. Under Assumption 1, after [25], the function $\dot{\bar{q}}: \mathbb{R}_{\geq 0} \rightarrow$ $\mathbb{R}^{N \times N}$ is persistently exciting.

Theorem 1 (Main result): Consider the system (8) in closed loop with (13) and

$$
\begin{aligned}
u_{\omega} & =-L \theta+L \bar{q}(t) \kappa(s, e)+\alpha \\
\dot{\alpha} & =-K_{\alpha} \alpha-K_{I} \omega+\dot{p} \kappa(s, e)
\end{aligned}
$$

where $K_{\alpha}$ and $K_{I}$ are introduced in (18) and $\kappa$ is defined in (16). Suppose further that $K_{d t}$ and $K_{p t}$ in (13) are diagonal positive definite and Assumption 1 holds. Then, the origin of the closed-loop state space, i.e, $(e, s, v, \tilde{\theta}, \omega, \alpha)=$ $(0,0,0,0,0,0)$ is uniformly globally asymptotically stable.

The proof of Theorem 1 relies on Lyapunov's direct method hence, it is constructive, but lengthy. Only the main guidelines are presented here; the complete proof may be consulted in [25]. 
A strict Lyapunov function for the closed-loop system is constructed using the following global change of coordinates:

$$
\begin{aligned}
& e_{\theta}:=\tilde{\theta}+\bar{q}(t) \kappa(s, e) \\
& e_{\omega}:=\omega+\dot{\bar{q}}(t) \kappa(s, e) \\
& e_{\alpha}:=\alpha+\ddot{\bar{q}} \kappa(s, e) .
\end{aligned}
$$

Next, to compact the notation, we define $X_{t}:=$ $\left[v^{\top} e^{\top} s^{\top}\right]^{\top} \in \mathbb{R}^{3 N}, X_{r}:=\left[e_{\theta}^{\top} e_{\omega}^{\top}, e_{\alpha}^{\top}\right]^{\top} \in \mathbb{R}^{3 N}, \bar{e}=$ $\operatorname{diag}\left[e_{i}\right], \bar{s}=\operatorname{diag}\left[s_{i}\right], \bar{e}_{\omega}=\operatorname{diag}\left[e_{\omega i}\right]$, and $\bar{\kappa}=\operatorname{diag}\left[\kappa_{i}\right]$. Thus, differentiating on both sides of all equations in (20) and using (8a), we obtain

$$
\dot{X}_{t}=\left[\begin{array}{ccc}
-K_{d t} & -K_{p t} & 0 \\
0 & 0 & \dot{\bar{q}} \bar{\kappa}-\bar{e}_{\omega} \\
0 & -\dot{\bar{q}} \bar{\kappa}+\bar{e}_{\omega} & 0
\end{array}\right] X_{t}+\left[\begin{array}{c}
0 \\
\Phi^{\top} \mathcal{L} \\
\Phi^{\perp \top} \mathcal{L}
\end{array}\right] \Phi v
$$

$\dot{X}_{r}=\left[\begin{array}{ccc}0 & I_{N} & 0 \\ -L & 0 & I_{N} \\ 0 & -K_{I} & -K_{\alpha}\end{array}\right] X_{r}+\left[\begin{array}{c}\bar{q} \\ \dot{\bar{q}} \\ \dot{\bar{q}}\end{array}\right]\left[\bar{e} \Phi^{\top} \mathcal{L}+\bar{s} \Phi^{\perp \top} \mathcal{L}\right] \Phi v$

Then, consider the set

$$
\mathcal{D}:=\left\{\left(X_{t}, X_{r}\right) \in \mathbb{R}^{6 N}:\left(X_{t}, L e_{\theta}, e_{\omega}, e_{\alpha}\right)=0\right\} .
$$

Note that in view of Lemma $1,\left(X_{t}, X_{r}\right) \in \mathcal{D}$ if and only if $(v, e, s, \theta, \omega, \alpha) \in \mathcal{S}$ where

$$
\mathcal{S}:=\left\{(v, e, s, \theta, \omega, \alpha) \in \mathbb{R}^{6 N}:(v, e, s, L \theta, \omega, \alpha)=0\right\}
$$

which, in view of the properties of $L$, implies that $\theta_{i}=\theta_{c}$ for all $i \leq N$. In other words, full consensus is achieved if $\mathcal{D}$ is uniformly globally asymptotically stable.

Remark 2: It is worth stressing that uniform global asymptotic stability of $\mathcal{D}$ or, equivalently, of the equilibrium $\{(v, e, s, \tilde{\theta}, \omega, \alpha)=(0,0,0,0,0,0)\}$, is a much stronger property than the consensus non-uniform convergence property $(v, e, s, \tilde{\theta}, \omega, \alpha) \rightarrow(0,0,0,0,0,0)$.

The starting point in the construction of a strict Lyapunov function for (21) is the function $V_{1}: \mathbb{R}^{3 N \times N} \times \mathbb{R}^{N} \rightarrow \mathbb{R}_{\geq 0}$ defined by

$$
V_{1}\left(X_{t}, \theta\right)=v^{\top} K_{p t}^{-1} v+z\left(X_{t}, \theta\right)^{\top} \mathcal{L} z\left(X_{t}, \theta\right)
$$

in which we emphasize the dependence of $z$ on $e, s$, and $\theta$ - see (9). In view of Lemma $1, V_{1}$ satisfies

$$
\frac{|e|^{2}+|s|^{2}}{\lambda_{N}(L)}+v^{\top} K_{p t}^{-1} v \leq V_{1}\left(X_{t}, \theta\right) \leq v^{\top} K_{p t}^{-1} v+\frac{|e|^{2}+|s|^{2}}{\lambda_{2}(L)}
$$

so it is positive-definite, decrescent, and radially unbounded in $X_{t}$. Furthermore, its total derivative along the trajectories generated by (8a), (12), and (17a) yields

$$
\dot{V}_{1}\left(X_{t}, \theta\right)=-2 v^{\top} K_{p t}^{-1} K_{d t} v .
$$

Next, we remark that the nominal part of the angular motion dynamics (21b) (that is, with $v=e=s=0$ ) is linear; this makes it natural to introduce the quadratic function

$V_{2}\left(X_{r}\right):=c_{2}\left[e_{\theta}^{\top} L e_{\theta}+e_{\omega}^{\top} e_{\omega}+e_{\alpha}^{\top} K_{I}^{-1} e_{\alpha}\right]+c_{5} e_{\omega}^{\top} e_{\alpha}+e_{\theta}^{\top} L e_{\omega}$, where $c_{2}$ and $c_{5}$ are positive constants to be defined such that $V_{2}$ be positive definite with respect $\left(e_{\alpha}, e_{\omega}, e_{\theta}\right)$ and, at the same time, the total derivative of $V_{2}$ along the trajectories of (21b) satisfy

$$
\begin{aligned}
& \dot{V}_{2}\left(X_{r}\right) \leq-Y_{2}\left(X_{r}\right)+\text { nl.t. } \\
& Y_{2}\left(X_{r}\right):=c_{2} e_{\alpha}^{\top} K_{I}^{-1} K_{\alpha} e_{\alpha}+c_{5} e_{\omega}^{\top} K_{I} e_{\omega}+e_{\theta}^{\top} L^{2} e_{\theta}
\end{aligned}
$$

where "nl.t." stands for diverse nonlinear terms of undefined sign $-c f$. [25].

Thirdly, we introduce a term whose derivative generates negative terms in the remaining closed-loop state variables, $e$ and $s$. To that end, as for instance in [26], for a bounded function $\psi: \mathbb{R}_{\geq 0} \rightarrow \mathbb{R}^{N}$, with $|\psi|_{\infty}=b_{\psi}>0$, we define

$$
\begin{aligned}
\bar{Q}_{\psi}(t) & :=\operatorname{diag}\left[Q_{\psi_{i}}(t)\right] \\
Q_{\psi_{i}}(t) & :=1+2 b_{\psi} T_{\psi}-\frac{2}{T_{\psi}} \int_{t}^{t+T_{\psi}} \int_{t}^{m} \psi_{i}(s)^{2} d s d m .
\end{aligned}
$$

The interest of this function is that it admits the bounds $1 \leq$ $Q_{\psi_{i}}<b_{Q i}:=1+2 b_{\psi} T_{\psi}$ and, furthermore,

$$
\dot{Q}_{\psi_{i}}=-\frac{2}{T_{\psi}} \int_{t}^{t+T_{\psi}} \psi_{i}(s)^{2} d s+2 \psi_{i}^{2}
$$

Moreover, if $\psi_{i}$ is persistently exciting with parameters $\mu_{\psi}$ and $T_{\psi}$, we obtain

$$
\dot{Q}_{\psi_{i}} \leq-\frac{2 \mu_{\psi}}{T_{\psi}}+2 \psi_{i}^{2} .
$$

Thus, in view of the latter and the persistency-of-excitation condition on $\dot{p}$, the function

$$
V_{3}\left(t, X_{t}\right):=\kappa(e, s)^{\top} \bar{Q}_{\dot{q}_{i}}(t) \kappa(e, s)
$$

where $\dot{q}_{i}$ is the $i$ th element in the diagonal of the persistently exciting function $\dot{\bar{q}}$ - see (18), is positive definite and radially unbounded in $(e, s)$ and its total derivative satisfies

$$
\dot{V}_{3} \leq-\frac{2 \mu_{\dot{q}}}{T_{\dot{q}}}|\kappa(e, s)|^{2}+\text { nl.t. }
$$

These computations suggest that a strict Lyapunov function for (21) should contain the functions $V_{1}, V_{2}$, and $V_{3}$. However, the system being highly nonlinear and time-varying, a simple linear combination of the latter does not suffice to dominate all the resulting high-order terms. For this, we rely on the methods of strictification of Lyapunov functions, laid in [27]. Lengthy computations show that the following is a strict Lyapunov function for (21) and that the set $\mathcal{D}$ is uniformly globally asymptotically stable — see [25] for a detailed computation of its derivative. Let $^{2}$

$$
\mathcal{V}\left(t, X_{t}, X_{r}\right)=W\left(t, X_{t}, X_{r}\right)+\rho_{1}\left(V_{1}\right) V_{2}\left(X_{r}\right)+\rho_{2}\left(V_{1}\right) V_{1}
$$

where

$$
\begin{aligned}
W= & \gamma\left(V_{1}\right) V_{1}+V_{1} V_{3}\left(t, X_{t}\right)+\alpha\left(V_{1}\right) e^{\top} v-c_{1} V_{1} e^{\top} \dot{\bar{q}} s \\
& +c_{1} b_{q} \lambda_{N}(L) V_{1}^{2}+\left(\lambda_{N}(L)+\left|K_{p t}\right|\right) \alpha\left(V_{1}\right) V_{1}, \\
\rho_{1}\left(V_{1}\right)= & \frac{2 \sigma\left(V_{1}\right)}{c_{5} \lambda_{m}\left(K_{I}\right)}\left[\alpha\left(V_{1}\right)+c_{1} b_{q} V_{1}\right]+1,
\end{aligned}
$$

${ }^{2}$ Not to cumbersome the notation futher we drop the arguments of $V_{1}$. 


$$
\begin{aligned}
\sigma\left(V_{1}\right)= & \max \left\{\frac{16 T c_{1} b_{q}}{\mu}, \frac{4 \lambda_{N}(L)\left|K_{d t}^{-1} K_{p t}\right| \alpha\left(V_{1}\right) V_{1}}{\gamma\left(V_{1}\right)}\right\}, \\
\alpha\left(V_{1}\right)= & 4 b_{q}^{2} \lambda_{N}(L) V_{1}^{2}\left|K_{p t}^{-1}\right|+4 c_{1} b_{q}^{2} \lambda_{N}(L)\left|K_{p t}^{-1}\right| V_{1}^{2} \\
& +\frac{4 c_{1}}{c_{4}}\left|\dot{\bar{q}}^{2} \Phi^{\perp \top} \mathcal{L} \Phi\right|_{\infty}^{2}\left|K_{d t}^{-1}\right| V_{1}+c_{1}^{2} c_{4} b_{q}^{2}\left|K_{p t}^{-1}\right|, \\
\gamma\left(V_{1}\right)= & 2 c_{4} V_{1}^{2} \lambda_{N}(L)\left|K_{d t}^{-1} K_{p t}\right|\left|\bar{Q}_{\dot{q}} \Phi^{\top} \mathcal{L} \Phi\right|_{\infty}^{2} \\
& +2 c_{4} V_{1}^{2} \lambda_{N}(L)\left|K_{d t}^{-1} K_{p t}\right|\left|\bar{Q}_{\dot{q}} \Phi^{\perp \top} \mathcal{L} \Phi\right|_{\infty}^{2} \\
& +\frac{\partial \alpha}{\partial V_{1}} V_{1}\left(\left|K_{p t}\right|+\lambda_{N}(L)\right)+\frac{c_{4}}{2} c_{1} V_{1} \\
& +2 \alpha\left(V_{1}\right)\left|\Phi^{\top} \mathcal{L} \Phi\right|_{\infty}\left|K_{d t}^{-1} K_{p t}\right| \\
& +\frac{c_{4}}{2}\left|K_{p t} K_{d t}^{-1}\right| \alpha^{2}\left(V_{1}\right)+\frac{c_{4}}{2} \alpha\left(V_{1}\right)\left|K_{d t}\right| \\
& +2 c_{1} b_{q} \lambda_{N}(L) V_{1}+\frac{4}{c_{4}} V_{1}^{2} \lambda_{N}(L)\left|K_{d t}^{-1}\right| \\
& +\frac{c_{4}}{2} c_{1}^{2}\left|K_{d t}^{-1} K_{p t}\right|\left|\dot{\bar{q}}^{2}\left(\Phi^{\top} \mathcal{L} \Phi\right)^{2}\right|_{\infty},
\end{aligned}
$$

and

$$
\rho_{2}\left(V_{1}\right)=c_{3}\left[V_{1} \rho_{1}\left(V_{1}\right) \lambda_{N}(L)\left[\left|\Phi^{\top} \mathcal{L} \Phi\right|_{\infty}+\left|\Phi^{\perp \top} \mathcal{L} \Phi\right|_{\infty}\right]\right] .
$$

An appropriate choice of $c_{1}$ and $c_{4}$ guarantees positive definiteness of $W$ with respect to $(e, s, v)$ - see [25] for details.

Remark 3: Note that $\left|\Phi^{\top} \mathcal{L} \Phi\right|_{\infty}$ is short-hand notation for the $\sup _{t>0}\left|\Phi(\theta(t))^{\top} \mathcal{L} \Phi(\theta(t))\right|$ which is bounded since $\Phi(\theta)$ is uniformly bounded.

Furthermore, the constants $c_{1}, c_{2}, c_{3}$ and $c_{4}$ are:

$$
\begin{aligned}
c_{1}= & 1+\frac{\lambda_{N}(L)}{\max \left\{2, \frac{2 T}{\mu}\left(1+\frac{2 N}{\lambda_{2}(L)}\right)\right\}}, \\
c_{2}= & 4 c_{5} \lambda\left(K_{I} K_{\alpha}\right)+4 c_{5} \lambda_{M}\left(K_{I}^{2} K_{\alpha}\right)+4 c_{5}^{2} \lambda_{M}\left(K_{I} K_{\alpha}^{-1}\right) \\
& +4 \lambda_{M}\left(K_{I} K_{\alpha}^{-1}\right)+2 \lambda_{n}(L)+4+2 \lambda_{M}\left(K_{I}\right) c_{5}^{2}, \\
c_{3}= & 2 b_{q}^{2}\left[\left(2 c_{2}+1\right)^{2}+\frac{\lambda_{M}\left(K_{I}^{-1}\right)}{c_{5}}\left(2 c_{2}+c_{5}+\lambda_{n}(L)\right)^{2}+\right. \\
& \left.\frac{\lambda_{M}\left(K_{\alpha}^{-1} K_{I}\right)}{c_{2}}\left(2 \lambda_{M}\left(K_{I}^{-1}\right)+c_{5}\right)^{2}\right]\left|K_{d t}^{-1} K_{p t}\right|, \\
c_{4}= & \max \left\{2, \frac{2 T}{\mu}\left(2+\frac{8 N}{\lambda_{2}(L)}\right)\right\}, \\
c_{5}= & 4 \lambda_{n}(L) \lambda_{M}\left(K_{I}^{-1}\right) .
\end{aligned}
$$

Since $\rho_{1}$ and $\rho_{2}$ are positive functions and radially unbounded, positive definiteness of $\mathcal{V}$ with respect to $\mathcal{D}$ is ensured under the proposed parameters $c_{i}$ with $i \in\{1, \ldots, 5\}$. Indeed, $V_{1}$ is positive definite with respect to $(e, s, v), W$ is so with respect to $(e, s, v)$, and $V_{2}$ is also so with respect to $\left(e_{\alpha}, e_{\theta}, e_{\omega}\right)-$ see [25].

Lengthy, but straightforward computations that involve the definitions of $\rho_{1}, \rho_{2}, \sigma, \gamma$ and $c_{i}$ above, lead to the expression

$$
\begin{aligned}
\dot{\mathcal{V}} \leq & -\frac{\rho_{1}\left(V_{1}\right)}{8}\left[c_{2} e_{\alpha}^{\top} K_{I}^{-1} K_{\alpha} e_{\alpha}+c_{5} e_{\omega}^{\top} K_{I} e_{\omega}+e_{\theta}^{\top} L^{2} e_{\theta}\right] \\
& -\frac{1}{4} \gamma\left(V_{1}\right) v^{\top} K_{d t} K_{p t}^{-1} v-\frac{1}{8} \alpha\left(V_{1}\right) e^{\top} K_{p t} e-\frac{\mu}{4 T} V_{1}^{3}
\end{aligned}
$$

—see [25] for details. The result follows.

\section{Simulations}

In this section we present some illustrative numerical simulations using four differential wheeled mobile robots. The desired formation pattern is fixed to be a rhomboid with an interconnection graph that has the following Laplacian matrix

$$
L=\left[\begin{array}{rrrr}
1 & -1 & 0 & 0 \\
-1 & 2 & 0 & -1 \\
0 & 0 & 1 & -1 \\
0 & -1 & -1 & 2
\end{array}\right] \text {. }
$$

The initial positions and the relative distances with regards to the barycentre of the formation are given in Table I. For sake of space, we only show simulations of the full consensus controller. The control gains have been set to: $K_{d t}=3 \mathbf{I}$;

TABLE I

INITIAL CONDITIONS AND RELATIVE DESIRED POSITIONS.

\begin{tabular}{c|c|c|c|c|c|}
\cline { 2 - 6 } & $x_{i}(0)$ & $y_{i}(0)$ & $\theta_{i}(0)$ & $\delta_{x_{i}}$ & $\delta_{y_{i}}$ \\
\hline 1 & 5 & -7 & $-(1 / 3) \pi$ & -2 & 0 \\
\hline 2 & -4 & 7 & $(2 / 3) \pi$ & 0 & 2 \\
\hline 3 & -2 & -5 & $(4 / 3) \pi$ & 2 & 0 \\
\hline 4 & 7 & 3 & 0 & 0 & -2 \\
\hline
\end{tabular}

$K_{p t}=5 \mathbf{I} ; K_{d t}=3 \mathbf{I} ; K_{\alpha}=2 \mathbf{I}$ and $K_{I}=\mathbf{I}$. The persistently exciting function $p(t)$ is given by $p(t)=\sin (0.1 t)$. The results are illustrated in Figs. 1-3.

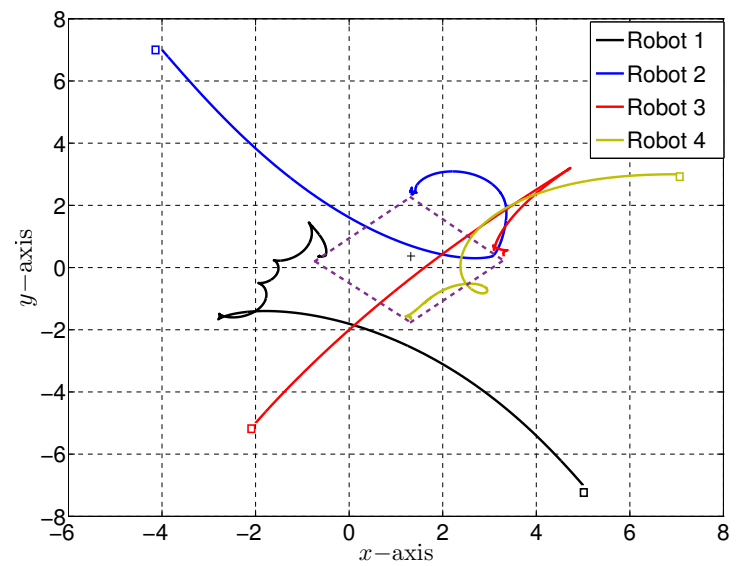

Fig. 1. Trajectories and formation of the network of mobile robots.

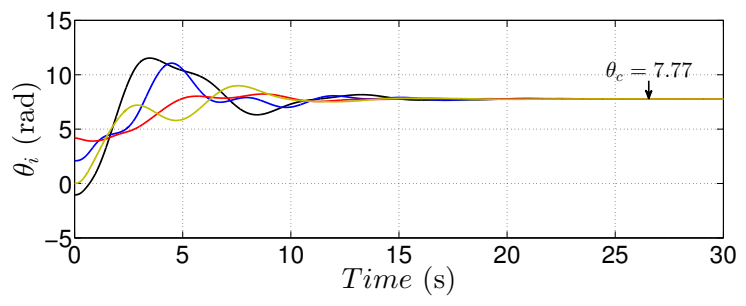

Fig. 2. Orientation of each mobile robot in the network

Fig. 1 shows the $x_{i}, y_{i}$ trajectories of the nonholonomic mobile robots, while Figs. 3 and 2 depict the relative positions $z_{i}$ and the orientation of the robots. From these plots it can be observed that the robots reach the desired formation and they agree on their final orientation. From the plot in Figure 1 one may notice a slightly oscillatory behaviour of the robots before 
reaching the consensus posture. This is a rather typical performance of persistency-of-excitation based controllers, which has been compared in [32] to that of dicontinuous set-point controllers for individual velocity-controlled robots.
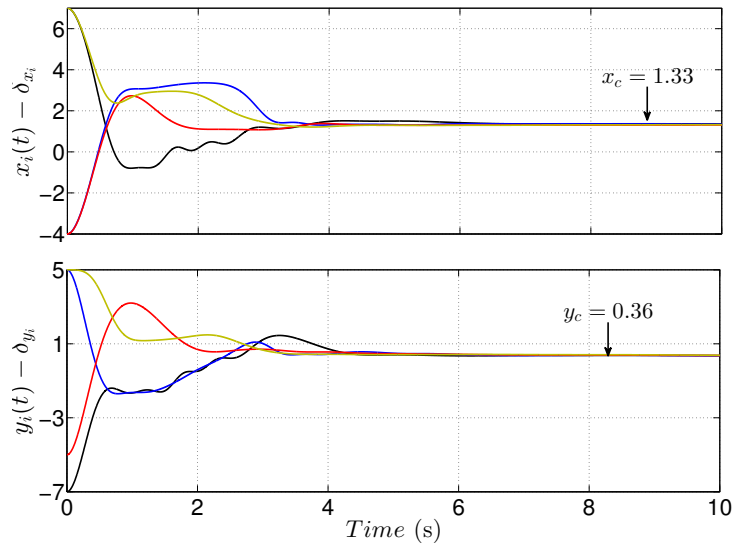

Fig. 3. Evolution of the relative positions $z_{i}$.

\section{CONCLusions}

A solution to the full-consensus problem of autonomous vehicles based on a complete kinematics-dynamics model and a smooth time-varying controller has been presented. For the first time in the literature we have provided a strict Lyapunov function to establish the main result. Beyond the undoubted benefits of establishing uniform global asymptotic stability, having a strict Lyapunov function is the first step towards important relaxations in our setting, as for instance, the consideration of an environment that includes obstacles. This topic is currently under research.

\section{REFERENCES}

[1] R. R. Olfati-Saber and R. M. Murray, "Consensus problems in networks of agents with switching topology and time-delays," IEEE Transactions on Automatic Control, vol. 49, no. 9, pp. 1520-1533, 2004.

[2] W. Ren, R. W. Beard, and E. M. Atkins, "A survey of consensus problems in multi-agent coordination," in Proc. of the American Control Conference, pp. 1859-1864, IEEE, 2005.

[3] E. Kranakis, D. Krizanc, and S. Rajsbaum, "Mobile agent rendezvous: A survey," in Structural Information and Communication Complexity, pp. 1-9, Springer, 2006.

[4] Q. Hui, "Finite-time rendezvous algorithms for mobile autonomous agents," IEEE Transactions on Automatic Control, vol. 56, no. 1, pp. 207-211, 2011.

[5] E. Nuño, R. Ortega, L. Basañez, and D. Hill, "Synchronization of networks of nonidentical Euler-Lagrange systems with uncertain parameters and communication delays," IEEE Transactions on Automatic Control, vol. 56, no. 4, pp. 935-941, 2011.

[6] E. Nuño, I. Sarras, and L. Basañez, "Consensus in networks of nonidentical Euler-Lagrange systems using $\mathrm{P}+\mathrm{d}$ controllers," IEEE Transactions on Robotics, vol. 26, no. 6, pp. 1503-1508, 2013.

[7] E. Panteley, A. Loría, and A. E. Ati, "On the analysis and control design for networked stuart-landau oscillators with applications to neuronal populations," in Proc. of the 54th. IEEE Conf. on Decision Control, 2015.

[8] J. Cortés, S. Martínez, and F. Bullo, "Robust rendezvous for mobile autonomous agents via proximity graphs in arbitrary dimensions," IEEE Transactions on Automatic Control, vol. 51, no. 8, pp. 1289-1298, 2006.

[9] L. Moreau, "Stability of continuous-time distributed consensus algorithms," in Proc. of the 43rd. IEEE Conf. on Decision and Control, vol. 4, pp. 3998-4003, 2004.
[10] G. Ferrari-Trecate, M. Egerstedt, A. Buffa, and M. Ji, "Laplacian sheep: A hybrid, stop-go policy for leader-based containment control," in Hybrid Systems: Computation and Control, pp. 212-226, Springer, 2006.

[11] G. Lafferriere, A. Williams, J. Caughman, and J. Veerman, "Decentralized control of vehicle formations," Systems \& control letters, vol. 54, no. 9, pp. 899-910, 2005.

[12] R. Olfati-Saber, "Flocking for multi-agent dynamic systems: Algorithms and theory," IEEE Transactions on Automatic Control, vol. 51, no. 3, pp. 401-420, 2006.

[13] Z. Lin, B. Francis, and M. Maggiore, "Necessary and sufficient graphical conditions for formation control of unicycles," IEEE Transactions on Automatic Control, vol. 50, no. 1, pp. 121-127, 2005.

[14] D. V. Dimarogonas and K. J. Kyriakopoulos, "On the rendezvous problem for multiple nonholonomic agents," IEEE Transactions on Automatic Control, vol. 52, no. 5, pp. 916-922, 2007.

[15] Z. Peng, G. Wen, A. Rahmani, and Y. Yu, "Distributed consensusbased formation control for multiple nonholonomic mobile robots with a specified reference trajectory," International Journal of Systems Science, vol. 46, no. 8, pp. 1447-1457, 2015.

[16] A. Bautista-Castillo, C. Lopez-Franco, and E. Nuno, "Consensus-based formation control for multiple nonholonomic robots," in Accepted in 2016 IEEE International Autumn Meeting on Power, Electronics and Computing (ROPEC), IEEE, 2016.

[17] C. Yang, W. Xie, C. Lei, and B. Ma, "Smooth time-varying formation control of multiple nonholonomic agents," in Proc. of the 2015 Chinese Intelligent Systems Conference, pp. 283-291, Springer, 2016.

[18] W. Dong and J. A. Farrell, "Consensus of multiple nonholonomic systems," in Proc. of the 47th IEEE Conf. on Decision and Control, pp. 2270-2275, IEEE, 2008.

[19] A. Ajorlou, M. Asadi, A. G. Aghdam, and S. Blouin, "Distributed consensus control of unicycle agents in the presence of external disturbances," Systems \& Control Letters, vol. 82, pp. 86-90, 2015.

[20] H. A. Poonawala, A. C. Satici, H. Eckert, and M. W. Spong, "Collisionfree formation control with decentralized connectivity preservation for nonholonomic-wheeled mobile robots," IEEE Transactions on Control of Network Systems, vol. 2, pp. 122-130, June 2015.

[21] A. Loría, E. Panteley, and A. Teel, "A new persistency-of-excitation condition for UGAS of NLTV systems: Application to stabilization of nonholonomic systems," in Proc. of the 5th. European Control Conf., (Karlsrühe, Germany), pp. 1363-1368, 1999.

[22] A. Loría, E. Panteley, and K. Melhem, "UGAS of skew-symmetric timevarying systems: application to stabilization of chained form systems," European Journal of Control, vol. 8, no. 1, pp. 33-43, 2002.

[23] Y. Wang, Z. Miao, H. Zhong, and Q. Pan, "Simultaneous stabilization and tracking of nonholonomic mobile robots: A lyapunov-based approach," IEEE Transactions on Control Systems Technology, vol. 23, pp. 1440-1450, July 2015.

[24] M. Maghenem, A. Bautista-Castillo, E. Nuño, A. Loría, and E. Panteley, "Consensus-based formation control of nonholonomic robots using a strict Lyapunov function," IFAC-PapersOnLine, vol. 50, no. 1, pp. 24392444, 2017. Presented at IFAC World Congress 2017, Toulouse, France. DOI: $10.1016 /$ j.ifacol.2017.08.406.

[25] M. Maghenem, Stability and stabilization of networked varying systems. PhD thesis, Univ Paris Saclay, Gif sur Yvette, 2017. Available online at: https://tel.archives-ouvertes.fr/tel-01596158/document.

[26] F. Mazenc, "Strict Lyapunov functions for time-varying systems," $\mathrm{Au}$ tomatica, vol. 39, no. 2, pp. 349-353, 2003.

[27] M. Malisoff and F. Mazenc, Constructions of Strict Lyapunov functions. London: Springer Verlag, 2009.

[28] K. D. Do, Z.-P. Jiang, and J. Pan, "A global output-feedback controller for simultaneous tracking and stabilization of unicycle-type mobile robots," IEEE Transactions on Robotics and Automation, vol. 20, no. 3, pp. 589-594, 2004.

[29] M. Maghenem, A. Loría, and E. Panteley, "A robust $\delta$-persistently exciting controller for leader-follower tracking-agreement of multiple vehicles," European Journal of Control, vol. 40, pp. 1-12, 2018. Appeared online: sept. 2017. DOI: 10.1016/j.ejcon.2017.09.001.

[30] M. Maghenem and A. Loría, "Strict Lyapunov functions for time-varying systems with persistency of excitation," Automatica, vol. 78, pp. 274 $279,2017$.

[31] A. Loría, E. Panteley, D. Popović, and A. Teel, "A nested Matrosov theorem and persistency of excitation for uniform convergence in stable non-autonomous systems," IEEE Transactions on Automatic Control, vol. 50, no. 2, pp. 183-198, 2005.

[32] B. Kim and P. Tsiotras, "Controllers for unicycle-type wheeled robots: Theoretical results and experimental validation," IEEE Transactions on Robotics and Automation, vol. 18, no. 3, pp. 294-307, 2002. 